Volume 9, No.4, July - August 2020

International Journal of Advanced Trends in Computer Science and Engineering

Available Online at http://www.warse.org/IJATCSE/static/pdf/file/ijatcse277942020.pdf

https://doi.org/10.30534/ijatcse/2020/277942020

\title{
Evaluation of Communication Technologies for Household Security System
}

\author{
WooLim Kim ${ }^{1}$, Sang Boem Lim ${ }^{2, *}$ \\ ${ }^{1}$ Department of Smart ICT Convergence, Konkuk University, Seoul, Korea, kw1930521@konkuk.ac.kr ${ }^{2}$, \\ $2^{2 *}$ Department of Smart ICT Convergence, Konkuk University, Seoul, Korea, sblim@konkuk.ac.kr
}

('corresponding author)

\begin{abstract}
As the number of single-person households increased, the difficulties and worries of single-person households are becoming big issues of our society. In order to protect single-person households, we are proposing a household security system. This household security system needs communication services to exchange photos, videos, voice, and text with visitor. For smooth communication between the Raspberry Pi and the smart phone, a total of two communication services, Bluetooth and Wi-Fi. We also present development environment of our system.
\end{abstract}

Key words : Bluetooth, Household Security system, raspberry pi, Wi-Fi

\section{INTRODUCTION}

As the number of single-person households increased, the difficulties and worries of single-person households are becoming big issues of our society. Table 1 lists the current concerns of single-person households. According to the Table 1 , single person who is age of $20 \mathrm{~s}$ and $30 \mathrm{~s}(40.8 \%$ and $46.7 \%)$ concerned mostly about their psychological stability, such as loneliness. Psychological stability issue is the most concerned matter to the single person household in overall. Health care is seen as the second most worrisome with 43.9 percent of the total. Especially, age of 40s and 50s (54.9\% and 50.2\%) are more concerned about health care. Safety and threats accounts for 31.2 percent of the total, accounting for more than 30 percent of those in their 20s, 30s and 40s, and are found to be of great concern to 37.1 percent, especially those in their $20 \mathrm{~s}$, of age. Other concerns were considered in meal resolution and stable job, residential and living environment, and the peripheral glance.

\footnotetext{
${ }^{1}$ This paper is based on the master thesis of WooLim Kim who is the first author of this paper at the Konkuk University.
}

Figure 1 illustrates the safety difficulties of a single person's life. The safety and threats of single-person households are divided into two categories, first priority and first and second priority combined. The results show that $37.8 \%$ of first priority and $55.1 \%$ of first and second priority combined of single person have difficulties with 'Emergency Paramedics'. The second most difficulty of single person household is the 'Residential intrusion safety' issue which is obtains $30.4 \%$ of first priority and $15.5 \%$ of first and second priority combined.

Table 1: A single-person household's current concern (\%) [1]

\begin{tabular}{|c|c|c|c|c|c|}
\hline & Total & 20s & $\mathbf{3 0 s}$ & $\mathbf{4 0 s}$ & $\mathbf{5 0 s}$ \\
\hline $\begin{array}{c}\text { Psychological } \\
\text { stability, such as } \\
\text { loneliness }\end{array}$ & 44.3 & 40.8 & 46.7 & 42.2 & 46.0 \\
\hline Health care & 43.9 & 32.5 & 35.6 & 54.9 & 50.2 \\
\hline Safety, Threats & 31.2 & 37.1 & 31.8 & 33.5 & 24.2 \\
\hline Meal resolution & 30.6 & 37.1 & 35.4 & 22.2 & 29.0 \\
\hline Stable job & 20.8 & 19.3 & 21.5 & 21.8 & 19.8 \\
\hline $\begin{array}{c}\text { Residential and } \\
\text { Living Environment }\end{array}$ & 16.5 & 25.0 & 16.8 & 13.7 & 13.3 \\
\hline Peripheral glance & 12.1 & 7.8 & 11.6 & 10.8 & 16.9 \\
\hline
\end{tabular}

In addition, the 'Safety of living careless' accounted for $27.7 \%$ of first priority and $8.2 \%$ of first and second priority combined, while the 'Burglary in one's absence' such as theft or theft issues accounted for $27.2 \%$ of first priority and $10.3 \%$ of first and second priority combined. Finally, 'The absence of a companion or protector, such as walking' accounted for $13.8 \%$ of first priority and $5.5 \%$ of first and second priority combined.

In this paper, we compare some communication technologies like Wi-Fi and Bluetooth, that are used to develop household security system. We also present development environment of our system. The goal of our system is to prevent concerns 
about the housing environment and housing safety of a growing number of single-person, hearing-impaired households and to improve the inconvenience of painful communication among the foreign visitors experienced by the weak, such as the popular phobia. This system also allows hearing-impaired people to recognize doorbell sounds quickly and simply and communicate smoothly with non-disabled visitors in order to relieve the discomfort experienced by hearing impaired people.

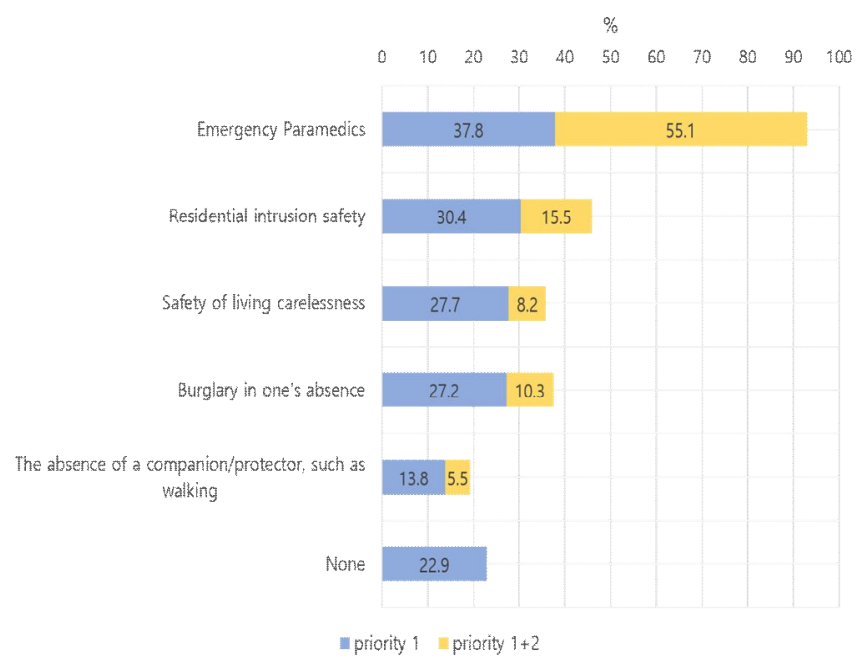

Figure 1: Safety difficulties in one's life [1]

\section{RELATED WORK}

Currently, various smart doorbells like Nest's Hello, Amazon's Ring and Xiaomi's Video doorbell are available on the market.

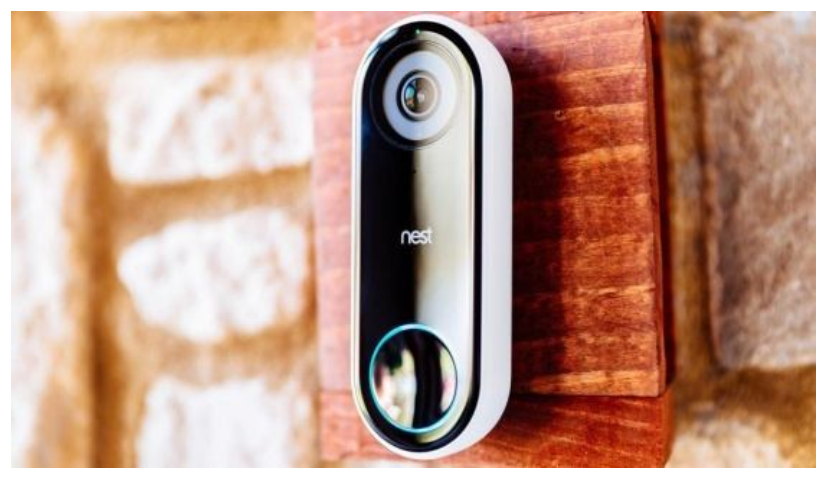

Figure 2: Nest Hello [2]

Nest's Hello (Figure 2) that is a smart video doorbell with built-in cameras, LEDs, and speakers, enable continuous video recording and recording through cameras and microphones. When a visitor clicks Hello, video information, along with a call signal, is immediately streamed to the owner's smart phone app. Users can check if visitors have come outside through the smart phone app and can also talk by pressing the microphone button on the app. With its motion sensing sensor Nest Cam IQ, users can detect visits by outside visitors, give motion and sound notification, and register family members or friends in advance through face recognition to identify who the visitors are without pressing the doorbell. In addition, infrared imaging is possible, allowing users to view external images even at night. It also has the ability to instantly identify visitors based on data from other users by applying cloud and artificial intelligence solutions. In addition, when a user is away from home, he or she can respond quickly to visitors through prerecorded messages. It is easy to record during a vacation because the cloud allows user to pay $\$ 5$ a month for data to be stored in the cloud, and if user pay extra money, user can increase the storage period. [2]

The Amazon's Ring (Figure 3) uses live video to transmit visitors' images to the smart phone, which can be seen immediately from outside through monitoring. When a person is away for a long time, he or she can automatically record the situation outside the house, and when a suspicious person is standing in front of the house, he or she can notify the alarm via his smart phone and report it to the police immediately. With Alexa, users can talk to visitors even if they are not at home and open doors remotely. It pays $\$ 10$ a month to record, store and share all the videos taken with a camera through the cloud. [3]

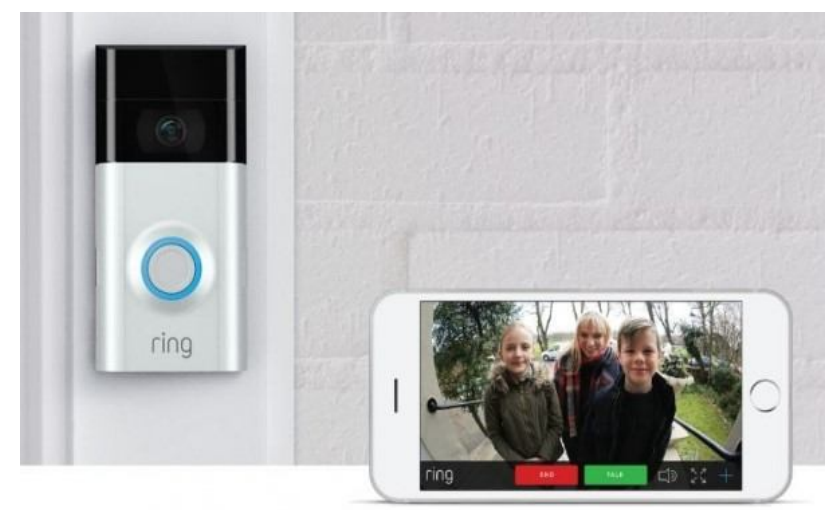

Figure 3: Amazon Ring [3]

Xiaomi's image doorbell (Figure 4) is equipped with a PIR human response sensor and takes pictures of movements within 3 meters of the door due to Motion Clip inspection and sends them to a smart phone. In addition, Xiaomi Ai facial recognition technology identifies and guides families and strangers. The IR-CUT built-in auto-transition air function allows the automatic switching of day and night mode according to the ambient light. Finally, it can be changed to a male voice through voice modulation. [4] 


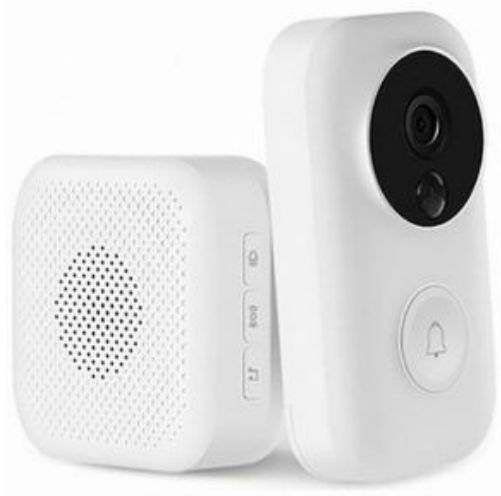

Figure 4: Xiaomi Image doorbell [4]

These products, however, do not communicate with the hearing-impaired claimed by the paper as a system in which hearing-impaired people can recognize visits by external visitors, but receive information by voice or video, no different from conventional doorbell. Use either SI (MKS) or CGS as primary units. (SI units are strongly encouraged.) English units may be used as secondary

\section{COMPARISON OF COMMUNICATION SERVICE}

\subsection{Wi-Fi and Bluetooth}

Wireless Fidelity (Wi-Fi) [5] is a trademark of Wi-Fi Alliance and is defined as a product that corresponds to a Wireless Local Area Network (WLAN) based on the Electronic Engineers Association (IEEE) 802.11 standard. Wi-Fi is a technology that allows access to wireless NFC networks within a certain distance from where the AccessPoint (AP) is installed, and $802.11 \mathrm{~b}$ or $802.11 \mathrm{~g}$ uses the $2.4 \mathrm{GHz}(12 \mathrm{~cm})$ and $5 \mathrm{GHz}(6 \mathrm{~cm})$ wireless bands. $\mathrm{Wi}-\mathrm{Fi}$ is aimed at making information accessible easily, enhancing compatibility and wirelessly accessing data and peripherals to avoid complexity. With Wi-Fi, wireless LAN can be used even where there is no network cable. Wi-Fi's communication distance is at least $50 \mathrm{~m}$ to $100 \mathrm{~m}$ from the point of access, and bandwidth and security are high. However, the use of Wi-Fi can be limited because of this, as well as high power consumption.

Bluetooth [5] that is developed by the Bluetooth Technology Multinational Alliance (SIG), is an NFC device that connects and exchanges information with NFC devices in a communication standard designed with short distances and low power based on transceiver microchips. The communication distance of Bluetooth is a class 2 device that is mostly used in smart phones and laptops, with a minimum range of 5 meters to 10 meters. Bluetooth can communicate with each other when each device is within a communication distance. There is a total of four classes in Bluetooth, compared to Class 2 devices with an output of $4 \mathrm{dBm}$ and a communication distance of 10 meters, while Class 1 devices have an output of $20 \mathrm{dBm}$ and a communication range of 100 meters. Therefore, the distance between Class 1 and Class 2 devices is much greater than when the same Class 2 devices communicate with each other. Bluetooth uses a method called "Frequency Hopping," in which multiple devices communicate at 79 frequencies by cutting off data transmitted without operating at fixed frequencies to prevent radio interference caused by using the same frequency band. Bluetooth uses $2.4 \mathrm{GHz}$, the frequency band of the Industrial Scientificand Medica (ISM), which can be used in various places, such as industry, science, and medical devices, and has low bandwidth and low security.

Table 2 compares Wi-Fi to Bluetooth. Of the two communication services, Wi-Fi and Bluetooth, Wi-Fi, which provides a wider range of communication and higher service in bandwidth and frequency ranges, is used as a smart doorbell communication service.

Table 2: Voice Service Comparison

\begin{tabular}{|c|c|c|}
\hline & Wi-Fi & Bluetooth \\
\hline Communication distance & $100 \mathrm{~m}$ & $10 \mathrm{~m}$ \\
\hline Operating frequency & $2.4 \mathrm{GHz}, 5 \mathrm{GHz}$ & $2.4 \mathrm{GHz}$ \\
\hline Bandwidth & High & Low \\
\hline Security & High & Low \\
\hline
\end{tabular}

\subsection{Port Forwarding}

A port is essential to operate multiple TCP/IP programs on a single computer. It can often see additional numeric addresses after an IP address, which is the port. Because the router's home gateway has Network Address Translation (NAT) capabilities, one external authorized IP is shared by all computers and all PC in the network are not private IP addresses on the external Internet. The DHCP server feature on the router automatically assigns the internal IP to each computer, which enables communication between each computer within the same network, and for Internet access, the computer's internal private I P is connected to an external authorized IP to communicate and connect.

From the outside, all computers connected to the network appear to have an authorized IP, so they are safe because there is no risk of attack. However, when certain computers need to be connected for certain purposes, such as FTP, they have internal IP, which creates an inaccessible problem. Port forwarding is a mechanism that is created as a way to address this [6]. Port forwarding refers to the process of intercepting information and data in the middle and redirecting it to 
another location while data is being transferred between computers on the local network and computers on the external network. Transferring a user-specified port to another computer allows the port associated with the specified port to access data from the local computer that was transferred [6].

\subsection{Data Transmission and Reception Test}

In this paper, we test data transmission rate with voice and text file with Wi-Fi and Bluetooth communication service to determine which communication service is suitable to our system environment.

Table 3 shows the average time that data have been exchanged. Voice and text are divided into three categories: 'Yes' for the shortest sentence, 'Thank you' and 'Package' for the middle sentences, and 'Leave it there' for the longest sentences in Korean language. The total transfer time is measured in milliseconds by checking the time that a file is transferred and the time it is received.

Table 3: Average time to send and receive data

\begin{tabular}{|c|c|c|c|}
\hline $\begin{array}{l}\text { Servic } \\
\text { e }\end{array}$ & Size & Time & \\
\hline \multirow{3}{*}{ Voice } & $\begin{array}{c}411.4 \mathrm{~KB} \\
(1.1 \mathrm{~s})\end{array}$ & $1.375 \mathrm{~s}$ & Yes, sir \\
\hline & $\begin{array}{c}424.8 \mathrm{~KB} \\
(2.1 \mathrm{~s})\end{array}$ & $1.376 \mathrm{~s}$ & Delivery \\
\hline & $\begin{array}{c}438.9 \mathrm{~KB} \\
(3.1 \mathrm{~s})\end{array}$ & $1.418 \mathrm{~s}$ & Please leave it there \\
\hline \multirow{3}{*}{ Text } & 3 Byte & $0.122 \mathrm{~s}$ & Yes, sir \\
\hline & 15 Byte & $0.134 \mathrm{~s}$ & Thank you \\
\hline & 28 Byte & $0.136 \mathrm{~s}$ & Please leave it there \\
\hline
\end{tabular}

The transfer is used an FTP library and this test is tested 1,000 times per file per service size. The Wi-Fi network has an operating frequency of $2.4 \mathrm{GHz}$ and a protocol of $802.11 \mathrm{n}$, with internet speeds fluctuating between 1.1 Mbps and 2.8 Mbps at the time of the test.

Comparing the transmission time of voice and text files show that text data can be sent and received in less than a second without significant error in any size. The result of text data transmission illustrate that the smallest 3Byte file can be transferred in 0.12 seconds, while the 15 Byte file and 28 Byte file take about 0.13 seconds. In the case of voice data, the transmission time take longer than the text data. The result of voice data transmission illustrate that the smallest 411.4 Kbyte file take about 1.1 seconds, the 423.4 Kbyte file take about 2 seconds, and the voice file of 436.8 Kbyte take about 3 seconds.

Considering the difference in transfer time between text file and voice file for one second and the Speed to Text (STT) and Text to Speed (TTS) conversion time, we decide to use text data with STT and TTS to communicate between raspberry pi and smart phone. STT and TTS conversion take place in raspberry pi. Based on the data transmission test in Table 4, we identify the time when we run STT and TTS with only text files.

Table 4 shows that the transfer time of a text file and the conversion time of STT and TTS. For STT, the three sentences - 'Yes,' 'Thank you,' and 'Leave there' - are recognized then calculated the time to be stored as text, and for TTS the time to recognize the text file and to be output as voice.

Table 4: Doorbell transfer time (seconds)

\begin{tabular}{|c|c|c|c|c|}
\hline & Size & $\begin{array}{c}\text { Transfer } \\
\text { time }\end{array}$ & STT & TTS \\
\hline \multirow{4}{*}{ Text } & 3 Byte & 0.122 & 0.00093 & 2.51 \\
\cline { 2 - 5 } & 15 Byte & 0.134 & 0.00094 & 2.78 \\
\cline { 2 - 5 } & 28 Byte & 0.136 & 0.00123 & 3.14 \\
\hline
\end{tabular}

The results show that STT make textual sentences almost real-time with spoken language. The 3 Byte file is created at 0.00093 seconds, 0.00094 seconds for the 15 Byte file, and 0.00123 seconds for the 28 Byte file. On the other hand, TTS takes much longer to create from text file to speech file. Even the smallest text file ( 3 Bytes) has a delay of about 2.51 seconds, 2.78 seconds for the 15 Byte file, and 3.14 seconds for the 28 Byte file.

STT uses a microphone connected to the raspberry pi to recognize the voice in real time from the networked Google Cloud engine, to convert directly to text and save the converted text to a file. TTS take a lot of time compared to STT because the text file is first sent to the Google Cloud engine, then converted the text file to a voice file, and then printed through a speaker connected to the raspberry pi. In the future, we also will evaluate voice response system like [7] to extend our system. 


\section{DEVELOPMENT ENVIRONMENT}

\subsection{Raspberry Pi Environment}

The smart doorbell is operating all functions through raspberry pi. Raspberry Pi [8] is a low-cost single board computer designed by the Raspberry Pi Foundation for basic computer science education and can be used as an external memory to install various Linux operating systems such as Devian-based Raspbian, Ubuntu, Kail Linux, and Arch Linux to establish a stable system. Constructors and circuit diagrams of the manufactured doorbell species used an open source project to create fabrication circuits for electronic hardware design.

To give a more intuitive and simpler view of the design interface that connects the surrounding components to the raspberry pi, we draw a circuit diagram of the smart doorbell species. Figure 5 depicts the final connection using raspberry pi and components as the expected circuitry of the smart doorbell species proposed in this paper. Smart doorbell consist of Raspberry Pi3B+, the main body of the doorbell implementation, a bread board that allows users to easily configure electronic circuits to make prototypes without soldering to connect raspberry pi and sensors, a button sensor that tells users that an external visitor has been received with the doorbell buttons, a raspberry pi camera module V2, a speaker AU connection to accept the voice of visitors.

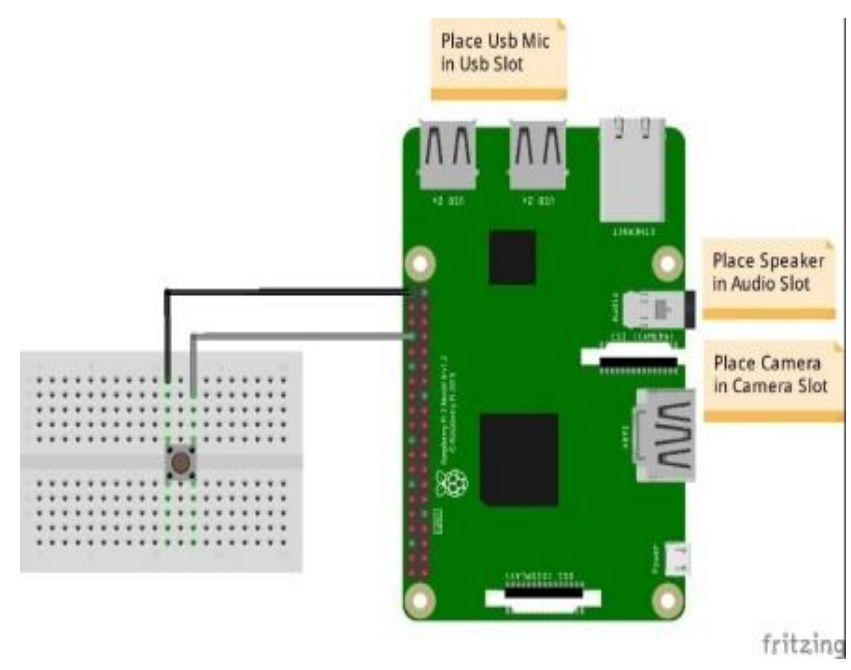

Figure 5: Smart doorbell circuit diagram

First of all, the raspberry pi's internal logic operates at $3.3 \mathrm{~V}$ and has a total of 40 pin numbers. The 40 pins are separated by General-purpose input/output (GPIO) and GND (GND), which are digital signal pins used as port pins for user control of behavior such as inputs and outputs. Digital pins are represented by a single bit and can only have a value of 0 or 1 , 0 is connected to the GND at $0 \mathrm{~V}$ and 1 is connected to the GPIO pin number that the user wants to connect to at $3.3 \mathrm{~V}$. In addition, the V2 camera, the USB microphone, and the AUX speaker are connected to each camera slot, USB slot, and audio slot built into the raspberry pi body.
The raspberry pi has two connectors that can connect flat cables, located in the center of the board and on the end of the board with a slot that says CAMERA and a slot that says DISPLAY. The V2 camera's cable is a flex cable, exposing a metal surface that can transmit electrical signals on one side and covered on the other with film. The Raspberry Pi's CAMERA connector also consists of an electrical circuit capable of transmitting electrical signals. The cable and connectors can be fitted and inserted into the CAMERA slot so that the electrical signals from the V2 camera and the raspberry pi can be transmitted.

We are using a microphone that is a USB round microphone that could be connected with a USB terminal that does not require extra equipment, and the speaker also connected to an AUX terminal that can be connected without additional equipment. We would like to learn more about the specs of the raspberry pi Camera Module V2, which have complex specs, unlike the surrounding components that have the specs of Raspberry Pi, the main body of the smart doorbell species, and easy and simple specs.

Table 5: Raspberry pi spec [9]

\begin{tabular}{|c|c|}
\hline \multicolumn{2}{|c|}{ Specifications } \\
\hline Introduction Date & $3 / 14 / 2018$ \\
\hline CPU & BCM2837B0 \\
\hline SoC & Quad Cortex-A53@ 1.4GHz \\
\hline Instruction set & ARMv8 \\
\hline RAM & MB SDRAM \\
\hline Storage & Gigabit \\
\hline Ethernet & WLAN/Bluetooth 4.2 \\
\hline Wireless & HDMI/Composite \\
\hline Video Output & HDMI/Headphone \\
\hline Audio Output & 40 \\
\hline GPIO & \\
\hline
\end{tabular}

Table 5 is a table of specifications for raspberry pi to be used in implementing smart doorbell species. The raspberry pi 3B+ was launched on March 14, 2018 and is equipped with the Cortex-A53 (ARMv8) series, which has a clock speed of 64 bits and $1.4 \mathrm{GHz}$, an updated version of Broadcom's application processor as the main CPU. The raspberry pi has $1 \mathrm{~GB}$ of LPDDR2 SDRAM and has a built-in Micro-SD slot in the raspberry pi to add storage space to store data. Raspberry $\mathrm{Pi}$ is equipped with a wireless LAN with $802.11 \mathrm{~b} / \mathrm{g} / \mathrm{n} / \mathrm{ac}$ at $2.4 \mathrm{GHz}$ and $5 \mathrm{GHz}$, Bluetooth 4.2 and Gigabit Ethernet with a maximum throughput of 300Mbps. CSI camera ports for connecting raspberry pi cameras and DSI ports for connecting raspberry pi displays are supported, as well as USB and AUX connectors in addition to HDMI. A $5 \mathrm{~V} / 2 \mathrm{~A}$ adapter is available for power supply. 
Table 6: Raspberry pi OS

\begin{tabular}{|c|c|}
\hline \multicolumn{2}{|c|}{ Specifications } \\
\hline NAME & Raspbian GNU/Linux \\
\hline VERSION & 10 (buster) \\
\hline HOME_URL & http://www.raspbian.org/ \\
\hline
\end{tabular}

Table 6 is a table of specifications for the OS installed inside the raspberry pi. Raspberry Pi OS is installed in Micro-SD and can be operated on Raspberry Pi. The Raspberry Pi OS used in this paper supports 10 Buster version with Devian Linux-based Raspbian, which is officially released in Raspberry Pi. The Raspbian OS is available for installation on Raspberry Pi's official website.

Table 7: Camera Specification [10]

\begin{tabular}{|c|c|}
\hline \multicolumn{2}{|r|}{ Specifications } \\
\hline Image Sensor & $\begin{array}{c}\text { Sony IMX } 219 \text { PQ CMOS image sensor } \\
\text { in a fixed-focus module. }\end{array}$ \\
\hline Resolution & 8-megapixel \\
\hline $\begin{array}{l}\text { Still picture } \\
\text { resolution }\end{array}$ & $3280 \times 2464$ \\
\hline $\begin{array}{l}\text { Max image } \\
\text { transfer rate }\end{array}$ & $\begin{array}{l}\text { 1080p: 30fps (encode and decode) } \\
\text { 720p: 60fps }\end{array}$ \\
\hline $\begin{array}{l}\text { Connection to } \\
\text { Raspberry Pi }\end{array}$ & $\begin{array}{l}\text { 15-pin ribbon cable, to the dedicated } \\
\text { 15-pin MIPI Camera Serial Interface } \\
\text { (CSI-2). }\end{array}$ \\
\hline $\begin{array}{l}\text { Image control } \\
\text { functions }\end{array}$ & $\begin{array}{c}\text { Automatic exposure control } \\
\text { Automatic white balance } \\
\text { Automatic band filter } \\
\text { Automatic } 50 / 60 \mathrm{~Hz} \text { luminance } \\
\text { detection } \\
\text { Automatic black level calibration }\end{array}$ \\
\hline Temp range & $\begin{array}{l}\text { Operating: }-20^{\circ} \text { to } 60^{\circ} \\
\text { Stable image: }-20^{\circ} \text { to } 60^{\circ}\end{array}$ \\
\hline Lens size & $1 / 4 ”$ \\
\hline Dimensions & $23.86 \times 25 \times 9 \mathrm{~mm}$ \\
\hline Weight & $3 g$ \\
\hline
\end{tabular}

Table 7 shows a specification of the raspberry pi camera module for real-time viewing of external visitors. The raspberry pi Camera V2 is officially launched by the Raspberry Pi Foundation and is available in a variety of ways, with a very small size of $23.86 \mathrm{~mm} \times 25 \mathrm{~mm} \times 9 \mathrm{~mm}$ and a light weight of $3 \mathrm{~g}$. The camera lens uses an 8-megapixel Sony IMX 219 image sensor.

Stopped images such as photos provide up to $3280 \times 2464$ pixels of performance, while moving images such as videos provide $30 \mathrm{fps}$ at $1080 \mathrm{p}$ resolution and $60 \mathrm{fps}$ at $720 \mathrm{p}$. It is possible to connect to and use the CSI camera port located in the center of the raspberry pi body as described in Table 7.

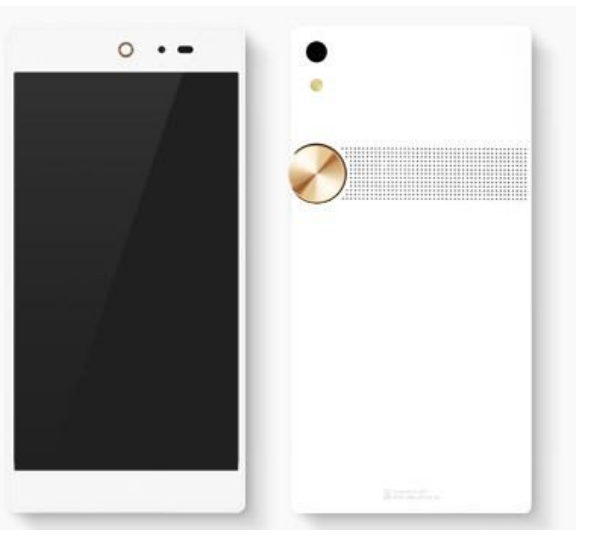

Figure 6: Mobile Device Environment

\subsection{Mobile environment}

Android studio are used to implement applications for smart interracial systems. Android is an operating system for smart phones made by Google and uses the Linux-based Java language. Android Studio is a program to develop applications in Android environments, and applications implemented used the Android Studio SDK 23 version.

Figure 6 used the Pantech company's IM 100 as a device used to implement the users. The processor of IM-100 is Quilcom's Snap Dragon 430 MSM8937 and is equipped with ARM Cortex-A53 and Qualcomm Adreno 505 with 8 -Core/1.4GHz clock speed. The memory is 2GB of LPDDR3 SDRAM and storage space provides $32 \mathrm{~GB}$ of internal memory and a Micro-SD slot that supports up to $2 \mathrm{~TB}$. It supports wireless LANs and Bluetooth 4.2(LEs) that support $5 \mathrm{GHz}$ of $802.11 \mathrm{~b} / \mathrm{g} / \mathrm{n} / \mathrm{ac}$ as well as LTE networks and NFC and GPS. The IM-100's operating system is Android-based Android 6.0.1 marshmallow with a 5.15" FHD display and a 3,000mAh internal battery of lithium ions. [11]

\section{REFERENCES}

1. Management institute Single-person Research Center, 2018 Korea Single-person Report, KB Financial Group, 2019. 06 
2. Nest. Google Nest Hello Doorbell, https://nest.com/doorbell/nest-hello/ (Accessed on Jul. 21, 2020)

3. Amazon. Ring Video Doorbell, https://ring.com/ Accessed on Jul. 20, 2020)

4. Xiaomi. Xiaomi Smart Video Doorbell, https://www.mi.com/global (Accessed on Jul. 30, 2020)

5. K. Pothuganti and A. Chitneni. A comparative study of wireless protocols: Bluetooth, UWB, ZigBee, and Wi-Fi, Advance in Electronic and Electric Engineering, Vol. 4, No. 6, pp. 655-662, Sep. 2014.

6. K. Andreas, Port Forwarding - What is it \& How Can it Help You in 2020? Port Forwarding Definition \& Functions,

https://id.wizcase.com/blog/port-forwarding-apa-itu-ma nfaatnya-bagi-anda/ (Accessed on Aug. 5, 2020)

7. A. Coskun-Setirek and Z. Tanrikulu, Intelligent Interactive Voice Response Systems and Customer Satisfaction, International Journal of Advanced Trends in Computer Science and Engineering, Volume 8 No. 1, Feb. 2019.

https://doi.org/10.30534/ijatcse/2019/02812019

8. V. Osamor, O. Emebo, B. Fori, and M. Adewale. Engineering and Deploying a Cheap Recognition Security System on a Raspberry Pi Platform for a rural Settlement, International Journal of Advanced Trends in Computer Science and Engineering, Volume 8 No. 6, Jun. 2019. https://doi.org/10.30534/ijatcse/2019/36862019

9. Raspberry Pi Foundation, Raspberry Pi 3 Model B+, Raspberry pi https://www.raspberrypi.org/ (Accessed on Aug. 4, 2020)

10. RS Components Ltd., Raspberry Pi Camera V2 Camera Module Camera Module, https://ae.rsdelivers.com/ (Accessed on Aug. 6, 2020)

11. SKY, Pantech IM-100S Specification, https://www.devicespecifications.com/en/model/3d6b3c c6 (Accessed on Aug. 5, 2020) 